Lymphology 52 (2019) 177-186

\title{
MELILOTUS, RUTIN AND BROMELAIN IN PRIMARY AND SECONDARY LYMPHEDEMA
}

\author{
S. Michelini, A. Fiorentino, M. Cardone
}

Ospedale San Giovanni Battista - ACISMOM - Rome, Italy

\section{ABSTRACT}

As reported in the literature, benzopyrones (alpha and gamma) have important effects on the microcirculation through various mechanisms. Coumarins are an alpha-benzopyrone as derivatives of Melilotus Officinalis, while bioflavonoids are a gamma-benzopyrone and include Rutin. Alpha-benzopyrones have two fundamental pharmacological effects: they have pro-lymphokinetic action by activating contractility of lymphangions; and the activation of macrophages to provide a proteolytic effect. Gamma-benzopyrones, such as Rutin, have an important anti-exuding and membrane stabilizing effect. Bromelain is known for its anti-inflammatory effect. The present study enrolled 52 patients with primary and/or secondary lymphedema in clinical stages I or II (according to the ISL classification) with 31 cases involving the lower limbs and 21 cases involving the upper limbs. All subjects were given for six months a natural compound consisting of $100 \mathrm{mg}$ of natural Melilotus, that contains 20 grams of Coumarin, $300 \mathrm{mg}$ of Rutin and $100 \mathrm{mg}$ of Bromelain. The following parameters were studied at zero time (T0), after three months (T1), and after six months of treatment (T2): pitting, Stemmer's sign, measurement of limb circumferences, measurement of superficial tissue thickness in the affected limbs using ultrasound, and blood tests to evaluate hepatic function (ALT, AST, GGT, total and fractional bilirubin). At the end of the treatment (T2), the following results were observed: disappearance of pitting in $72 \%$ of the cases; unchanged Stemmer's sign; average decrease in limb circumferences of $4.2 \mathrm{~cm}$; and average reduction of the superficial thickness of 29\%. There was no variation in the liver function parameters examined. The combination of natural compounds (Melilotus, Rutin, and Bromelain) has been shown to be a valuable aid in the clinical control of both primary and secondary lymphedema of clinical stages I and II as well as in control of inflammatory phenomena related to chronic stasis. There were no side effects and no alteration of liver function parameters found.

Keywords: medical therapy of lymphedema, benzopyrones, Melilotus, Rutin, Bromelain

In normal conditions, tissue fluid is collected from the initial lymphatic vessels (simple anatomic structures formed by a single layer of endothelial cells, without basal membrane, permeable to interstitial contents) (1-3). From a physiological point of view, the lymphatic drainage depends on two fundamental factors: the increase in interstitial pressure (caused by muscular contractions and external compression) and the propulsive forc- 
es, associated with contraction and relaxation of the muscular component of the lymphatic collector's wall. According to the guidelines of the International Society of Lymphology, lymphedema is defined as a manifestation of an insufficiency of the lymphatic system or an imbalance in the lymph transport; it may be an isolated phenomenon or associated with a multitude of other local disabling sequels or even fatal systemic syndromes. The common denominator is that the lymphatic transport falls below the capacity required to manage the load of the microvascular filtrate, including the plasma proteins and the cells that normally leak out of the blood into the interstitium. This process culminates in excessive proliferation and deposition of substances in the extracellular (interstitial) matrix of the parenchymal and stromal elements $(1,2,4)$.

Primary lymphedema when familial is generally an autosomal dominant congenital disorder with incomplete penetrance $(5,6)$. The most frequently involved genes which are documented well in the literature, are FLT4 (alias VEGFR3; vascular endothelial growth factor 3) and FOXC2. Onset of clinical edema can occur at birth or immediately after (congenital), within 15-20 years of age (early), or after 20 years of age (late, the most frequent). Among the secondary forms, the most common, outside endemic areas for filariasis, are surgical causes in which tumor excision is associated with loco-regional lymphadenectomy. The onset time of secondary lymphedema varies, from 1-2 weeks up to 30 years.

The current international classification comprises four clinical stages: the first stage (stage 0), refers to a latent subclinical condition without evident edema, although lymphatic transport is compromised (positive lymphoscintigraphy). Stage 1 represents an edema with a high protein content compared, for example, with venous stasis edema and disappears with elevation of the limb. Pitting can be positive. In the next stage (Stage 2), distal edema is present and pitting is positive. In the more advanced phase, a small proportion of fibrotic tissue is possible. Limb elephantia- sis, dystrophic skin lesions and warts appear in Stage 3. Pitting is positive, and there may be acanthosis; tissue sclerosis and lipodystrophy have increased considerably.

Regarding the lower limb, the history of edema progression is very important. The Stemmer's sign is positive in the edema of the lower limbs (the examiner cannot raise a cutaneous fold of the toes from the underlying bone phalanx). Pain may be present or not (in the early stages of the disease, usually, it is absent).

The diagnosis of lymphedema can be obtained either by clinical or diagnostic examination. A detailed medical history and a complete physical examination, both general and local, are important. The Gold standard diagnostic exam, according to the guidelines of the International Society of Lymphology, is lymphoscintigraphy (1). The examination provides morphological and functional information on the lymphatic system, which several authors have confirmed since $1990(7,8)$. High resolution ultrasound allows to determine the localization of the edema in the suprafascial (lymphedema) or subfascial (phlebedema) compartment. In post-phlebitic syndrome, the consistency of the prevalent tissue (fluid, fibrous or mixed) can be used to monitor the effects of treatment on both the reduction of edema and the recovery of the muscular component (9). Computed Tomography better defines the position of edema, the prevalence of excess tissues, and provides important indications on muscles, bones and tendon components in order to better target physical therapy (10).

The essential treatment for lymphedema, both primary and secondary, is physical-rehabilitative treatment, associated with pharmacological and/or surgical therapy. The best therapeutic results, as in all the chronic disabling diseases, are obtained through a close collaboration between several health professionals involved in the rehabilitation project (11). The compliance of patients is essential for the acceptance of chronic treatment which is already difficult in the early stages of edema 
and becomes even more burdensome in the advanced stages.

Lymphedema is the only type of edema (other than inflammatory, high capillary permeability edema) with high interstitial protein concentration (high-protein fluid). In this sense, it differs from other forms of edema due to elevated venous pressure locally or systemically, in which the water portion prevails (hypo-protein fluid, which is generally easier to treat and drain).

There is a history in the literature of numerous studies on the efficacy of benzopyrones (12) to reduce both the water and protein portion of lymphedema, and most studies (since the '80s), show clinically significant results. A study by Grosso and Marra demonstrated that the combination of several benzopyrones increases its bio-availability and efficacy on the reduction of interstitial edema and on the modulation of the inflammatory activity, compared to each individual component even if administered at a higher dosage (13). Based on these data, we hypothesized the preferred use of a combination of Melilotus, Rutin, and Bromelain at concentrations reflecting the effective doses reported in the literature. The aim of this study was to evaluate the efficacy and tolerability of this combination in patients with primary or secondary lymphedema at stage I or II.

\section{MATERIALS AND METHODS}

52 consecutive patients were included ( 50 females and 2 males, aged between 14 and 70 years) with primary or secondary lymphedema of the upper and/or lower limbs, either unilateral or bilateral at stage I or II ISL clinical classification for a total of $\mathbf{3 1}$ lower limbs and 21 upper limbs (Fig. 1). Exclusion criteria included: 1) patients with trophic lesions (ulcers, pachydermia, verrucosis); 2) Patients with renal insufficiency (from stage II) or with severe hepatic insufficiency; 3 ) patients with severe heart failure (NYHA class: 2-4). Diagnostic examinations were performed including high-resolution ultrasound of soft tissues in all patients (both at the beginning and at the end of treatment) and in primary forms, segmental lymphoscintigraphy of the upper and/or lower limbs. Most patients had lymphedema for more than 10 years (34.6\%) with $13.5 \%$ for less than 6 months and $19.2 \%$ less than 2 years (Fig. 2). Studies were approved by the Institutional Review Board with informed consent.

Patients in the study were given a combination of Melilotus, Rutin, and Bromelain for a period of six months. The method used was the administration of one tablet per day containing $100 \mathrm{mg}$ of Melilotus (containing 20\% Coumarin), $300 \mathrm{mg}$ of Rutin and $100 \mathrm{mg}$ of Bromelain. Any therapies prior to the recruitment (even use of diuretics) were not modified either at the beginning or during treatment.

Patients were assessed at baseline (T0), after three months (T1), and after six months of treatment (T2) for the following parameters: pitting, Stemmer's sign, measurement of circumferences of the limbs, measurement of ultrasound thicknesses of the superficial tissues in the affected limbs, VAS, assessment of the patient's well-being through the SF12 questionnaire, and laboratory examinations (complete blood count, erythrocyte sedimentation rate, CRP, SGOT, SGPT, $\alpha$-GT, alkaline phosphatase, total and fractional bilirubin).

\section{RESULTS}

At T0 patients presented with either weakly expressed $(40.4 \%)$ or more fully expressed (59.6\%) pitting. At T2, average disappearance of pitting was observed in $72 \%$ of cases while $3.8 \%$ of cases still expressed full pitting (Fig. 3). The Stemmer's sign at T2 remained unchanged.

Circumference of the limbs decreased by an average of $4 \%$ at $\mathrm{T} 1$ and $6 \%$ at $\mathrm{T} 2 \mathrm{com}$ pared to $\mathrm{T} 0$, with a minimum decrease of $2 \%$ and a maximum of $12 \% .29 \%$ of patients had no circumference reduction at $\mathrm{T} 1$ and these patients presented an average reduction of $5 \%$ at T2 (Figs. 4,5). The standard deviation was 2.1 with $83 \%$ of patients included in its range. The reduction in the volume of the limb was 
52 patients divided by age groups

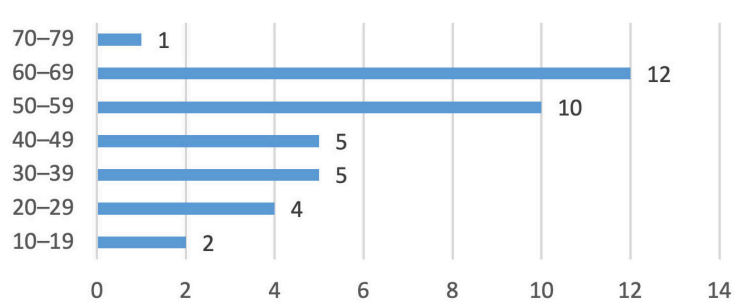

Distribution by sex

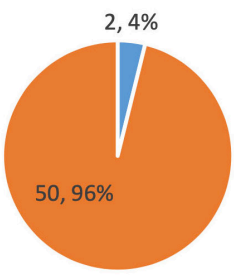

Fig. 1. Characteristics of patients in the study divided by age (left) and gender groups (right). Females in orange, males in blue.

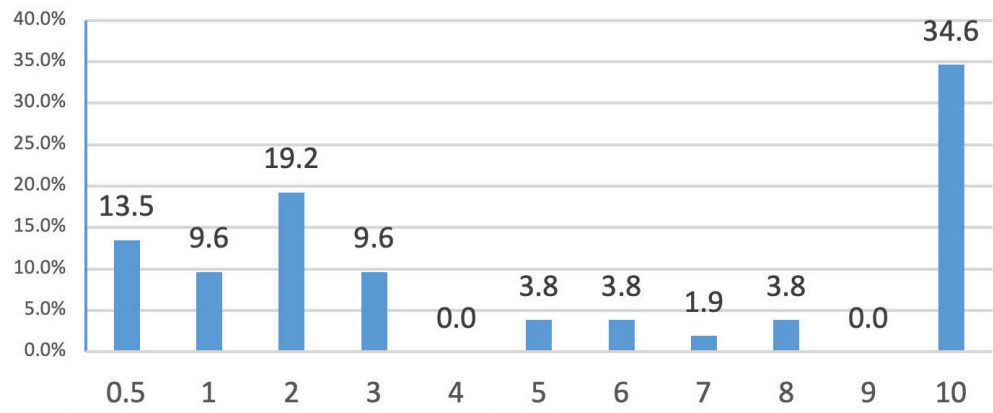

Fig. 2. Onset of lymphedema (years post) for patients included in the study.

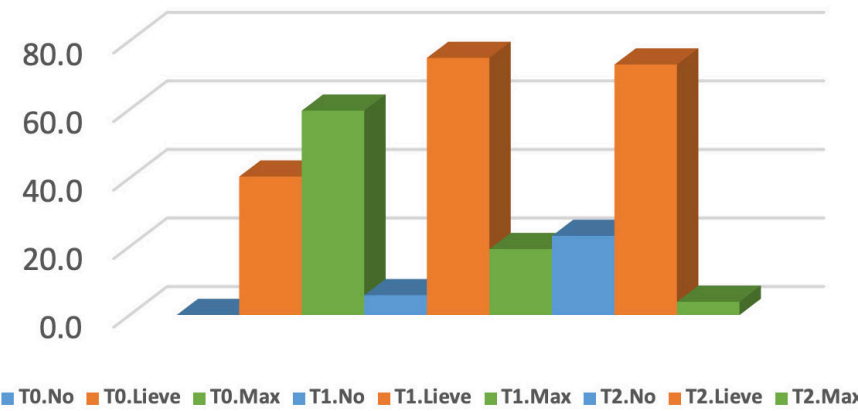

Fig. 3. Modifications of pitting scores in patients during the study period. $T 0=$ time point zero; $T 1=$ time point 1 ; $T 2=$ time point 2; No= no pitting; Light $=$ weakly expressed pitting; Max = fully expressed pitting.

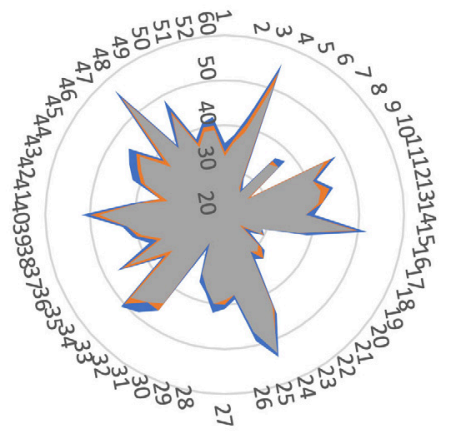

Fig. 4. Graphical depiction of circumference reduction (in cm) for all patients at T0 (blue), T1 (orange), and T2 (gray). significant $(\mathrm{p}<0.01)$.

Superficial thickness measured by ultrasound was decreased in all patients, except for one case (complicated by an episode of lymphangitis) where the superficial thickness was increased at $\mathrm{T} 2$. The average reduction was $8 \%(24 \mathrm{~mm})$ with a maximum of $26.4 \%$. $27 \%$ of patients had a reduction of more than 10\% (Fig. 6).

Perceived health status, assessed by the administration of the SF12 test, showed an 


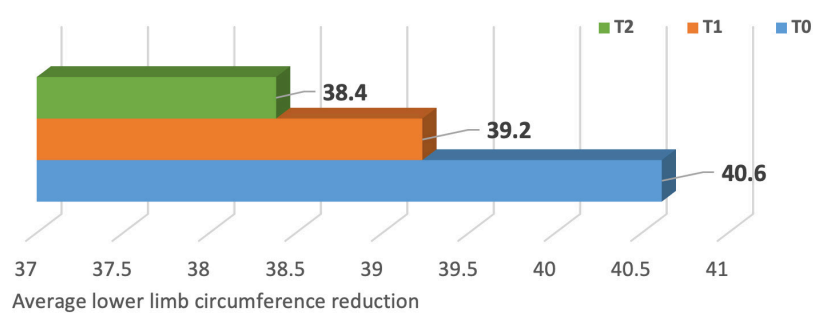

Circumference (in cm)

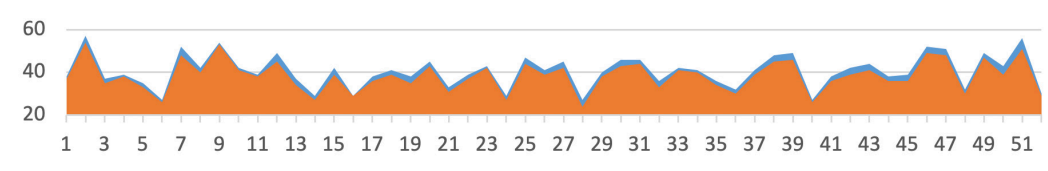

m Circ.Pre $\approx$ Circ.Post

Fig. 5. Average circumference reduction of the lower limbs. Top: average circumferences in cm at T0 (blue), T1 (orange), and T2 (green). Bottom: T0 circumference values (blue) overlaid by T2 (orange) circumference values for all patients in $\mathrm{cm}$.

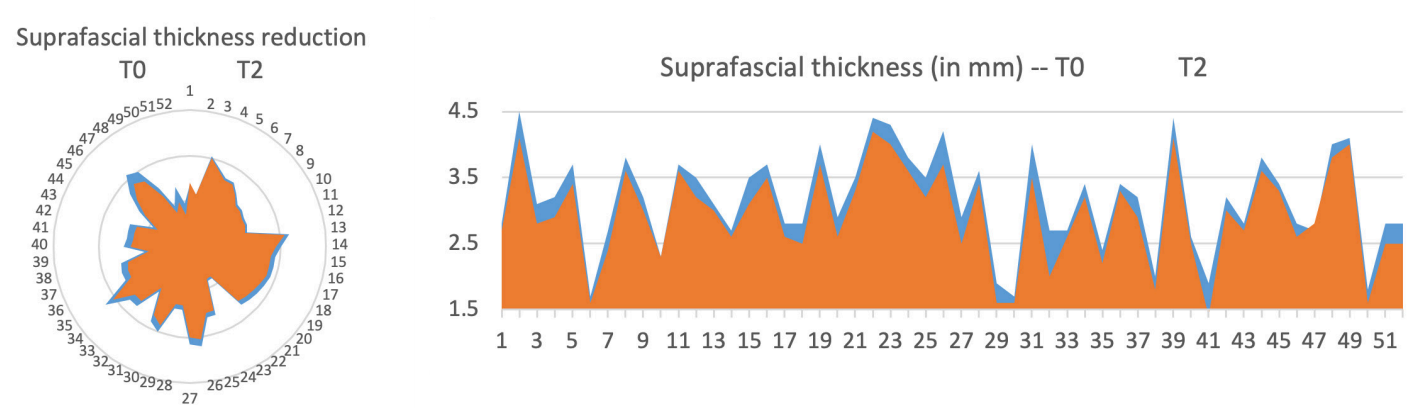

Fig. 6. Graphical depiction of the reduction in the suprafascial thickness at T2 (orange) expressed in mm respect to T0 baseline (blue) for all patients.

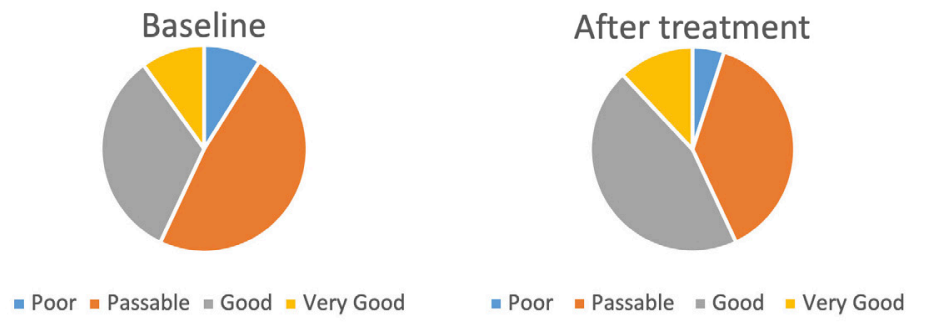

Fig. 7. Improved health status assessed by the SF12 test at baseline (left) compared to T2 (right).

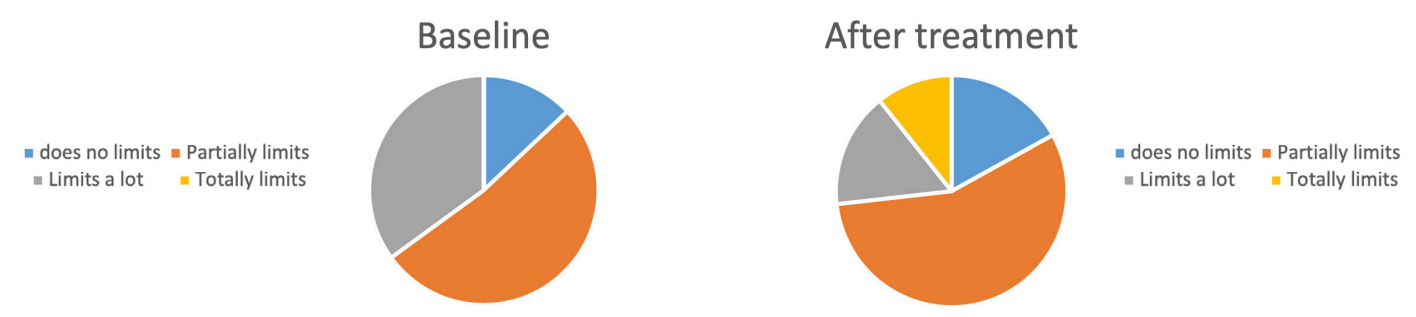

Fig. 8. Limitations in activities evaluated by the SF12 test comparing baseline (left) to T2 (right). 


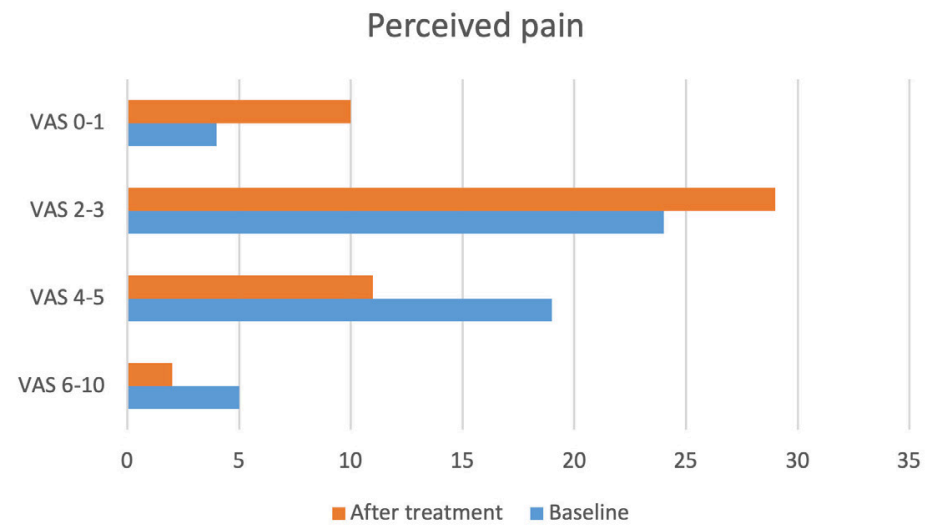

Fig. 9. Perceived pain results assessed by VAS scale demonstrate a decrease (shift to lower values) at time point T2 (orange) compared to baseline (blue).

increase of 'Good' value (29\%) and 'Very Good' value (45\%) at T2 compared to T0 with corresponding decrease in 'Poor' values (53\%) and 'Passable' values (34\%) at T2 (Fig. 7).

Similar behavior was observed by analyzing the "limitation in Activity" parameter of SF12 Test, which demonstrated increasing percentage of "Non-limiting" (52\%) and "Partially limits" (28\%) values with simultaneous decrease in "Limit a lot" (24\%) values compared to T0 (Fig. 8).

With regard to pain, understood as an element that hinders function, there was an increase in the values "None" $(31 \%)$ and "Very little" (26\%), associated with a decrease in "Slightly" (32\%) and "Very" (46\%) values at $\mathrm{T} 2$.

The administration of the analog VAS pain scale (values between 0 and 10) at T2 showed a decrease of $63 \%$ in subjects with pain with values reported between 6 and 10 and a decrease of $29 \%$ in subjects with pain between 4 and 6 . There was an increase of $65 \%$ of subjects with pain between 0 and 1 and $22 \%$ of those who reported pain between 2 and 4 (Fig. 9).

No substantial differences were observed for all the parameters examined between primary and secondary forms of lymphedema and no side effects or alterations of liver function parameters were found.

\section{DISCUSSION}

This prospective and observational study showed a good efficacy and tolerability of the combination of Melilotus, Rutin, and Bromelain in patients with primary and/or secondary lymphedema. We did not distinguish the results based on lymphedema etiology in the 52 patients studied, since the drugs affect the healthy interstitium, increasing its transport capacity, and do not claim to restore the disrupted lymphatic flow, both for the congenital and acquired forms.

A potential limitation is that this study lacked blinding. An additional limitation is the lack of a placebo group. We chose not to include a placebo group as we surmised that it would not have added any additional information from that obtained in the untreated population described in the literature.

The results on the efficacy of this study are encouraging for the use of phytotherapy in the control of lymphedema. The beneficial actions were also useful in the management of pain and symptoms related to edema, allowing patients to be more adherent to the medical, physical, and surgical therapies. This should allow better clinical outcomes and a significant increase in Quality of Life.

Examining the literature, it appears that one of the actions of the Melilotus extracts is to regularize and increase the contractions of 
the smooth musculature of the small lymphatic vessels. The lymphokinetic effect has been demonstrated by the increase in lymphatic return to the thoracic duct, found in both animal and man in treatment with this extract. The activity of reabsorption of lymphatic fluids is independent of the dosage, by saturation of the lymphocytic cell receptor involved in determining its therapeutic effect. In vitro studies (14) have shown that Melilotus extracts have a pronounced myotropic effect, stimulating the rhythm and the vasal tone. In vivo studies have shown that Melilotus extracts induce a marked increase in lymphatic flow in the thoracic duct with an average increase of $177 \%$ in dogs (15). A similar experiment on humans was performed by Bartoš and Brzek, who reported that intravenous administration of a preparation of Melilotus extracts in $\mathbf{1 0}$ volunteers induced an increase in lymphatic flow at the thoracic duct level of $96 \%$ after 10 minutes and an additional $35 \%$ after 20 minutes, confirming the lymphokinetic properties of Melilotus extracts (16).

The proteolytic action has been highlighted in other studies that demonstrate that the intake of alpha-benzopyrones causes a numerical increase of macrophages in the area of edema and increases the proteolytic activity with consequent removal of proteins from the interstitial spaces. Alpha-Benzo $\neg$ pyrones can reactivate macrophages, as demonstrated by in vitro studies on isolated macrophages. This precise proteolytic tissue action allows the reduction of both the concentration and the total quantity of tissue proteins. Casley-Smith demonstrated that proteolysis does not represent a significant phenomenon in healthy tissue but is needed to control the appearance and evolution of high-protein edema $(17,18)$. A series of studies on the pharmacodynamics of Melilotus extracts, including that of Zhou et al (19), have shown that Coumarin derivatives inactivate the nuclear factor NF- $\mathrm{kB}$, which stimulates the production of several pro-inflammatory cytokines (Interleukin- $\beta$, IL-6, TNF- $\alpha$ ) and other mediators (such as NO and COX2) $(19,20)$. At the same time, they reinforce the expression of anti-inflammatory factors, such as heme-oxygenase. The inhibition of NF- $\mathrm{KB}$ is linked to the blocking of the expression of other molecules, such as VEGF (Vascular Endothelial Growth Factor) and, therefore, to the regulation of microvascular permeability (21). Melilotus extracts can halve the expression of NF- $\mathrm{BB}$ and $\operatorname{VEGF}(22,23)$. As Nishikawa et al described, and subsequently confirmed and well framed by Liu et al, reduction of inflammatory edema and modulation of inflammation by Melilotus extracts showed an efficacy comparable to or greater than indomethacin $(24,25)$.

Pharmacokinetic studies in healthy volunteers have shown that Coumarin administered orally has a rapid gastrointestinal absorption (approximately 1 hour) which is almost complete (90\%). The effect of Coumarin persists for two weeks after the cessation of administration, confirming a receptor-like mechanism, which makes the target cells independent of the blood concentrations of the substance (buffer phenomenon) (26). The elimination of Coumarin and its metabolites is essentially urinary. Numerous studies on the clinical effects of Melilotus extracts have developed over the years; one of the most extensive revisions has been conducted by Casley-Smith, which considered 50 studies carried out with the administration of Melilotus both orally (38 studies) and topically (12 studies), singly or in combination with other phytotherapeutic treatments (in 11 studies/38 and 6 studies/12). The main result of the revision was the significant anti-edema effect of these preparations, with a significant decrease in edema from $17 \%$ to $55 \%$ of cases. Moreover, the extension of therapy to at least one year has brought a reduction of up to $78 \%$ (26). Casley-Smith carried out a placebo-controlled clinical trial that demonstrated the effect of Melilotus extracts on lymphedema following breast cancer surgery with a reduction in edema that was on average $40 \%$ in the group undergoing treatment with Melilotus, compared to placebo (28). In the literature it is reported that Melilotus Officinalis is very well tolerated. 
Occasionally mild gastrointestinal disturbances (nausea and vomiting) appear transiently and usually only at the beginning of treatment in a percentage of patients ranging from 1.5 to $5 \%$ of cases. In none of the cases treated with Melilotus extracts were alterations observed in the parameters of liver function or coagulation (29-31).

The optimal dose of Coumarin in Melilotus extracts is 20\%: 100 grams of natural Melilotus contains $20 \mathrm{mg}$ of Coumarin, the dose recommended by EFSA (European Food Safety Authority).

In various studies in the literature, the most studied and used of the gamma-benzopyrones is Rutin. In a study conducted in 1989 by Blumberg $\mathrm{S}$ et al, it has been shown that Rutin is able to reduce endothelial hyper-permeability induced until its normalization (21). Casley-Smith has shown that Rutin can reduce capillary filtration even in conditions of microcirculatory damage, studying changes in groups of patients with diabetic microangiopathy and venous hypertension (17). In addition, Rutin is a powerful scavenger of free oxygen radicals (ROS), which induce a series of adverse effects on the endothelium through different mechanisms such as lipid peroxidation, mitochondrial DNA damage and LDL oxidation (32). Rutin reduces and prevents the oxidative damage produced by free radicals. A significant work by Belcaro et al showed that Rutin was found to be effective in the control of signs and symptoms from chronic venous insufficiency and edema, also preventing complications of the pathologic conditions (33). The pineapple plant is rich in Bromelain, which is a set of various proteases (phosphatase, glucosidase, peroxidase, cellulase, protease inhibitors and endopeptidase). In general, Bromelain halves the time of removal of the protein portion in lymphatic edema and accelerates the healing time of soft tissues. The experimental evidence suggests a mechanism of action that can be traced back to this sequence: the increase of fibrinolytic activity and reduction of inflammation (antiplatelet effects and cellular antiadhesive) $\rightarrow$ reduction of vascular damage $\rightarrow$ increased micro-vasal permeability and removal of plasmatic residues. The anti-inflammatory activity, as suggested by Hotz et al, is linked to an interaction on the metabolism of eicosanoids, especially on lipoxygenase, and reduces the synthesis of inflammatory and vasoconstrictive substances: prostaglandins (PGE2) and thromboxanes (TBX2) (34-36). Fibrinolytic activity has been attributed to an increased conversion of plasminogen to plasmin, which depolymerizes fibrin and limits the diffusion of coagulative processes. The proteolytic activity, together with its debridement properties, can exert a "brush effect" on the extravasated proteins in the interstitium and can prevent the subsequent connective tissue organization with an inflammatory edema control, both post-surgical and primary. The beneficial actions of Bromelain have also been found to be useful in pain management and edema-related symptoms, making patients more adherent to the medical, physical and surgical therapies in the future (37-39).

To date, the association of supplements to physical therapy, which may precede or follow a possible surgical treatment, shows the best results with regard to the containment of edema and related symptoms $(40,41)$.

Moreover, the availability of preparations, which in single tablet (mono-administration) offer the effective concentrations of the benzopyrones studied, rather than using different products with the individual components, makes the therapy economically trackable in the long term by almost all of the patients.

The studies in the literature, associated with the clinical results obtained in this study with the improvement of the Quality of Life (with the possibility of reducing the costs borne by the patients) and the excellent tolerability, allow us to advise and extend the combined treatment of the studied phytotherapeutic preparation (Melilotus, Rutin, and Bromelain) with physical therapy to all patients with lymphedema (independent of its genesis), in order to obtain the best clinical result. Obviously, this does not provide healing from 
pathology, as no treatment currently available can do. In relation to tolerability, the control examinations have not shown any type of side effect even on liver and renal function, nor on myelopoiesis. The study was appropriately conducted on patients at stage I or II. In patients in stage III, tissue fibrosis is established at the local level that prevents the substances administered from being able to act. This is especially important for the pro-lymphokinetic effect, since in those anatomical conditions the wall of the lymphatic vessels is likely involved in a chronic fibrosclerotic process.

\section{CONCLUSIONS}

In the present study, the combination of Melilotus, Rutin, and Bromelain has been shown to offer valuable help in the clinical control of both primary and secondary lymphedema at clinical stage I or II and in the control of the inflammatory phenomena related to chronic lymph stasis. No evidence of unwanted side effects and no alteration of liver function parameters were found. Limitations of this study include independent confirmation of the botanical contents (i.e., benzopyrone content), relatively small size of the study, and lack of placebo controls.

This preliminary study shows significant clinical effects, which however, must be investigated in more numerous and more varied cases including from the point of view of the representativeness of both sexes and of the clinical localization.

\section{CONFLICT OF INTEREST AND DISCLO- SURE}

All Authors declare no competing financial interests exist.

\section{REFERENCES}

1. The Diagnosis and Treatment of Peripheral Lymphedema: 2016 Consensus Document of the International Society of Lymphology. Lymphology 49 (2016) 170-184.

2. Michelini, S, A Failla: Linfedemi: Inquadra- mento diagnostico clinico e strumentale. Minerva Cardioangiologica, 45 (Suppl. I al n ${ }^{\circ}$ 60 (1997), 11-15.

3. Földi, M, OT Zoltán: On the mechanism of action of a Melilotus preparation. Arzneimittelforschung 15 (1965), 899-901.

4. Allegra, C, M Bartolo Jr, R Sarcinella: Morphological and functional characters of the cutaneous lymphatics in primary lymphedemas. Europ. J. Lymphology Rel. Probl. 6 (1996), I24.

5. Lee, BB, M Andrade, PL Antignani, et al: Diagnosis and Treatment of Primary Lymphedema. Consensus Document of the International Union of Phlebology (IUP)-2013. International Angiology 32 (2013), 541-574.

6. Hariel, L, J Amir, M Nussinovitch, et al: Lymphedema praecox seen as isolated unilateral arm involvement: Case report and review of the literature. J. Pediatr. 130 (1997), 492-494.

7. Bourgeois, P, F Wolter: Lymphoscintigraphy demonstration of a protein losing enteropathy. Europ. J. Lymphol. Rel. Probl. 18 (1990), 4446.

8. Boccardo, F, C Bellini, C Campisi, et al: Role of lymphoscintigraphy in the indications to microsurgical treatment of peripheral lymphedema. Europ. J. Lymphology 16 (2006), 24-28.

9. Michelini, S, C Campisi, A Failla, et al: Proposal for stadiation of phlebolymphedema. Eur. J. Lymphol. Rel. Probl. 6 (1995), I-14.

10. Michelini, S, A Failla, GL Paroni Sterpini, et al: Limb plhebolimphedema: Diagnostic non invasive approach and therapeutical implications. Eur. J. Lymphology 5 (1995), 103-108.

11. Galli, T, R Sudati: Adequate post-surgery physiotherapy for women with breast cancer in evidence based medicine, Review. Eur. J. Lymphology 20 (2009), 11-16.

12. Bruneton, J: Pharmacognosie Phytochimie Des Plantes Medicinales. Lavoisier, Paris, 1993.

13. Grosso, A, M. Marra: Polifenoli, biodisponibilità e nutrizione, http://bio.uniroma2.it/polifenoli-biodisponibilita-e-nutrizione/, online pub, Istituto di Biologia Università di Tor Vergata.

14. Krajnovic, P: Lymphographic investigations concerning the influence of drugs on the lymphatic circulation. Arztl. Forsch. 23 (1969) 381-384.

15. Sandmann, W, V Herz: Risultati linfografici in seguito alla somministrazione di un prodotto a base di cumarina. Kreisl. 3 (1971), 124.

16. Bartoš, V, V Brzek: Effect of Esberiven forte on lymph flow in the human thoracic duct. Med. Klin. 65 (1970), 1701-1703.

17. Casley-Smith, JR, CT Wang, JR Casley-Smith, et al: Treatment of fibrosial lymphedema and elephantiasis with 5,6 benzo-alpha-pyrone, $\mathrm{Br}$. Med. J. 307 (1993), 1037-1041. 
18. Casley-Smith, JR: High Protein Edemas and the Benzopyrones. JB Lippincott Company, Sidney, 1986.

19. Zhou, Y, J Wang, W Yang, et al: Bergapten prevents lipopolysaccharide-induced inflammation in RAW264.7 cells through suppressing JAK/STAT activation and ROS production and increases the survival rate of mice after LPS challenge. Int. Immunopharmacol. 48 (2017), 159-168.

20. Neumayer, C, A Fügl, J Nanobashvili, et al: Combined enzymatic and antioxidative treatment reduces ischemia-reperfusion injury in rabbit skeletal muscle. J. Surg. Res. 133 (2006), 150-158.

21. Blumberg, S, G Clough, C Michel: Effects of hydroxyethyl rutosides upon the permeability of single capillaries in the frog mesentery. Br. J. Pharmacol. 96 (1989), 913-919.

22. Maksimovic', ZV, M Maksimivic', D Jadranin, et al: Medicamentous treatment of chronic venous insufficiency using semisynthetic diosmin - A prospective study, Acta Chir. Jugosl. 55 (2008), 53-59.

23. Aliev, SA, KG Ibishov, ES Aliev: Certain aspects of pathogenesis of hemorheogical and trophic disturbances and their pharmacological correction in patients with chronic venous insufficiency. Vestn. Khir. Im. I I Grek. 167 (2008), 39-43.

24. Nishikawa, M, A Yamashita, K Ando, et al: The suppressive effect of melilotus extract on the thermal edema of rats. Nihon Yakurigaku Zasshi. 3 (1983), 193-209.

25. Liu, YT, PH Gong, FQ Xiao, et al: Chemical Constituents and Antioxidant, Anti-Inflammatory and Anti-Tumor Activities of Melilotus officinalis (Linn.) Pall, Molecules 23 (2018). PII, E271, doi: 10.3390/molecules23020271.

26. Scondotto, G, D. Aloisi: Cumarine naturali nel trattamento dell'edema linfatico. Linfologia Oggi 1 (2002), 16-21.

27. Casley-Smith, JR: Benzo-pyrones in the treatment of lymphedema, Int Angiol. 18 (1999), 31-41.

28. Casley-Smith, JR, RG Morgan, NB Piller: Treatment of lymphedema of the arms and legs with 5,6-benzo-[alpha]-pyrone. N. Engl. J. Med. 329 (1993), 329, 1158-1163.

29. Abraham, K, F Wöhrlin, O Lindtner, et al: Toxicology and risk assessment of coumarin: Focus on human data. Mol. Nutr. Food Res. 54 (2010), 228-239.

30. Beinssen, APA: Possible coumarin hepatotoxicity. Med. J. Austr. 161, 725, 1994.
31. Cox, D, R O'Kennedy, RD Thomas: The rarity of liver toxicity in patients treated with coumarins. Hum. Toxicol. 8 (1989), 501-506.

32. Tondi, P: Terapia dell'insufficienza venosa cronica. Patologie del Sistema Venoso e Linfatico, Verduci Editore, Roma, 2013.

33. Belcaro, G, M Rosaria Cesarone, A Ledda, et al: O-(beta-hydroxyethyl)-rutosides systemic and local treatment in chronic venous disease and microangiopathy: An independent prospective comparative study. Angiology 59 (Suppl 1) (2008), 7S-13S.

34. Hotz, G, T Frank, J Zöller, et al: Antiphlogistic effect of bromelaine following third molar removal. Dtsch. Zahnarztl. Z. 44 (1989), 830-832.

35. Orsini, RA: Plastic Surgery Educational Foundation Technology Assessment Committee, Bromelain. Plast. Reconstr. Surg. 118 (2006), 1640-1644.

36. MacKay, D, AL Miller: Nutritional support for wound healing, Altern. Med. Rev. 8 (2003), 359-377.

37. Houck, JC, CM Chang, G Klein: Isolation of an effective debriding agent from the stems of pineapple plants. Int. J. Tissue Reactions 5 (1983), 125-134.

38. Singer, AJ, SA McClain, BR Taira, et al: Rapid and selective enzymatic debridement of porcine comb burns with bromelain-derived Debrase: Acute phase preservation of non injured tissue and zone of stasis. J. Burn Care Res. 31 (2010), 304-309.

39. $\mathrm{Hu}, \mathrm{W}, \mathrm{AM}$ Wang, SY Wu, et al: Debriding effect of bromela in on fire arm wounds in pigs. J. Trauma 71 (2011), 966-972.

40. Pastura, G, M Mesiti, M Saitta, et al: [Lymphedema of the upper extremity in patients operated for carcinoma of the breast: Clinical experience with coumarinic extract from Melilotus Officinalis.] Clin. Ter. 150 (1999), 403-408.

41. Casley-Smith, JR, Judith R Casley-Smith: Treatment of lymphedema by complex physical therapy with and without oral and topical benzopyrones: What should therapists and patients expect. Lymphology 29 (1996), 76-82.

\author{
Sandro Michelini, MD \\ Hospital San Giovanni Battista \\ Via Luigi Ercole Morselli 8 \\ Rome, ITALY 00148 \\ Telephone \#: 39 06/655961 \\ FAX \#: 39 06/65596235 \\ e-mail: sandro.michelini@fastwebnet.it
}

